\title{
Accessing integrated and nonintegrated propositional structures in memory
}

\author{
FRANK R. YEKOVICH \\ Catholic University of America, Washington, D.C. 20064 \\ and \\ LEON MANELIS \\ Illinois State University, Normal, Illinois 6176I
}

\begin{abstract}
This paper investigates the access properties associated with different propositional structures. Two memory experiments are reported, in which the underlying structures of sentences were integrated or not. Some sentences tested had the same concept repeated across the propositions (integrated), whereas other sentences had no explicit repeated arguments (nonintegrated). Accessibility to the memory traces of the sentences was manipulated through the acquisition and the testing conditions. In Experiment 1, subjects received either immediate or delayed recall tests, under free or cued conditions. Integrated sentences were recalled better than nonintegrated ones under conditions of high accessibility (immediate recall or delayed cued recall). In contrast, under the low-access condition (delayed free recall), nonintegrated sentences were recalled slightly better than the integrated ones. Experiment 2 confirmed and extended the results for delayed free recall. Here again, under conditions of low sentence access, nonintegrated sentences were recalled better. These results were interpreted according to theory dealing with the lag effect in list learning.
\end{abstract}

One aspect of recent work on the structure of text is the connectedness of the text's underlying propositions. The propositions can be connected by means of shared concepts. Using Kintsch's (1974) system, for example, the sentence "The enemy soldier fired the cannon" can be represented by two propositions, (FIRE, SOLDIER, CANNON) and (ENEMY, SOLDIER), which are connected through the shared concept, or "argument," SOLDIER. We will refer to sentences whose propositions are clearly connected in this way as "integrated," or Type A. In contrast, some sentences are not so clearly integrated. For example, "Soldiers fought and the bombs continued" can be represented by two propositions without shared arguments: (FIGHT, SOLDIER) and (CONTINUE, BOMB). Under some conditions subjects may connect these two propositions by adding a third proposition on their own, such as (HURL, SOLDIER, BOMB). Nevertheless, this type of sentence is not so explicitly integrated as the first type, and we will call it "nonintegrated," or Type B.

These types of sentences were tested in our earlier work on the facilitative effects of argument repetition

The research described here was a joint effort; order of authorship was determined by random assignment. We want to thank Carol Walker for her help with Experiment 1 and her critical reading of an earlier version of the paper. This work was partially supported by Illinois State University and by computer services at Catholic University. Send requests to Frank R. Yekovich, School of Education, Catholic University of America, Washington, D.C. 20064.
(Manelis \& Yekovich, 1976). In those experiments, reading time and free recall immediately after reading each sentence showed better performance for Type A sentences than for Type B sentences. Those findings were consistent with other research demonstrating better recall for organized or integrated material than for nonintegrated material (e.g., Hayes-Roth \& Thorndyke, 1979; Kintsch, 1974; Moeser, 1979; Thorndyke, 1977). However, in our earlier study (Manelis \& Yekovich, 1976) we also obtained a somewhat surprising result. In a delayed free recall test that occurred after all sentences had been presented, propositional recall was no longer better for Sentence Type A; instead, in two experiments, Type B sentences were recalled as well as or slightly better than the A sentences. Thus, there was a discrepancy between immediate and delayed recall-an apparent interaction between time of test and type of sentence. In that study the main concern was with immediate rather than delayed testing, and the experiments were not designed for well-controlled comparisons between those two testing conditions. Hence, the purposes of the present study were (1) to determine whether the prior discrepancy we observed incidentally was spurious or genuine, (2) to interpret the results according to some general principles of memory, and (3) to test the interpretation.

One theoretical interpretation for the possible interaction between time of test and sentence type can be based on the process of accessing a sentence in memory. In immediate recall, that is, recall immediately after 
reading a sentence, access to at least some part of a sentence seems virtually certain. Nevertheless, one factor limiting the level of recall would be the difficulty of retrieving other parts of the sentence from the part initially accessed. Given this recall situation, the integrated A sentences would be remembered better than the nonintegrated B sentences because of their explicitly connected propositional network. That is, because the memory structure for a Condition A sentence is integrated, once a component of the structure is accessed, retrieval of the remainder of it is enhanced relative to Type B. However, in delayed free recall, access to any sentence would not be so likely, and as a consequence, integrated sentences may no longer enjoy a recall advantage. The propositions for integrated sentences should be stored as a cluster in memory, whereas nonintegrated text should be represented by propositions at separate locations. During delayed free recall, it is possible that any one of several separately located propositions would more likely be found in memory than the clustered propositions of a connected structure. Thus, when overall access probability is low, Type B sentences may actually have a slight memory advantage over Type A sentences. In other words, the access factor might be enough to overcome the integration factor, thereby producing better performance for $B$.

Because of the potential importance of sentence access, we added cuing conditions to the present experiments. Cued recall or similar conditions, even with delayed testing, should make sentence access very likely, simulating immediate free recall. Thus, if our thinking about access is correct, A sentences should show better performance than $B$ at immediate recall (both free and cued) and at delayed cued recall, although B may prove better at delayed free recall.

In this report we describe two experiments that tested this access interpretation. Experiment 1 contrasted immediate and delayed testing, using free and cued retrieval conditions. Experiment 2 focused on delayed testing and compared standard learning conditions with an acquisition condition in which a key word in each sentence was underlined. Subsequent to the empirical presentation, we interpret our access findings within a framework that parallels recent theory on lag effects in word-list research (e.g., Glenberg, 1979).

\section{EXPERIMENT 1}

This experiment tested the access hypothesis by varying the type of recall testing conditions. The subjects were presented integrated (A) and nonintegrated (B) sentences and were then tested for sentence memory. There was also a third sentence condition containing extra arguments (C) that had been included in our original study (Manelis \& Yekovich, 1976). We included Condition $\mathrm{C}$ for two reasons. First, it allowed a complete replication of the immediate recall conditions of the earlier experiments (Manelis \& Yekovich, 1976). Second, if subjects can process these difficult sentences successfully, the extra propositional arguments might provide additional access points during retrieval, a possibility we noted in our earlier paper. Consequently, in delayed free recall, a slight memory superiority of $C$ over $B$ could occur. As in the earlier study, the present experiment utilized immediate and delayed recall. Immediate recall was defined as recall of a sentence immediately after its presentation. Delayed recall was sentence recall after the presentation of a block of sentences. Unlike our other study, however, time of test was orthogonally combined with type of test. Some subjects were given free recall tests of the sentences; other subjects were given cued recall. The recall prompts were single nouns that sometimes instantiated the underlying repeated argument.

According to our working hypothesis, recall for A sentences ought to exceed that of $B$ sentences when the probability of access is high. This result should occur in immediate testing, regardless of whether recall is free or cued. In delayed recall, however, the free and cued conditions should result in different levels of accessibility to the representations in memory. Delayed free recall is a condition of low accessibility and, as suggested by our earlier work, could result in an advantage for B. In delayed cued recall, however, the recall cues should facilitate access, thus again allowing a recall superiority for Condition A.

\section{Method}

Design. Four independent variables were combined factorially. Time of test (immediate, delayed) and type of recall (free, cued) varied between subjects. Sentence condition (A, B, C) and sentence length (four propositions, five propositions) were varied within subjects.

Materials and counterbalancing. Thirty-six sets of sentences were created. Each set contained three sentences, one in Condition $A$, one in $B$ and one in $C$. The sentences described short action sequences that followed in correct temporal and causal order. Sentences within each set were matched for general semantic content and also for number of propositions. Eighteen of the sets were constructed so that four propositions resulted in the text base of each sentence, and in the other 18 sets, there were five propositions for each sentence. Samples of the materials are provided in Table 1, along with the specifications for the sentences, based on Kintsch's (1974) system of analysis. Notice from the table that numbers of words and arguments were matched for Conditions A and B, while the number of shared arguments differed.

All of the sentences had grammatical constructions identical to those in the table of examples. In four proposition sentence sets, the subject noun of the first clause was identical across Conditions A, B, and C. For five proposition sets, the common noun of interest occurred as the first explicit subject in Condition $A$ and as the first noun in Conditions $B$ and $C$. This noun, common to the three sentence conditions, served as the repeated argument in the corresponding Condition $\mathrm{A}$ sentence. Further this noun represented the cue used for the cued recall tests.All but two of these recall prompts were animate, and none was a proper name.

Each subject read 36 experimental sentences: 12 As, 12 Bs, and $12 \mathrm{Cs}$. Half of the sentences in each condition were four propositions long and half were five. The 36 experimental 
Table 1

Example Sets of Sentences, Experiment 1

\begin{tabular}{|c|c|c|c|c|}
\hline & Words & Arguments & Repetitions & Sentence \\
\hline \multicolumn{5}{|c|}{ Four-Proposition Sentences } \\
\hline Condition A & $13(13.11)$ & $4(4)$ & $3(3)$ & $\begin{array}{l}\text { The farmer watched the clouds, smiled, held a hat, and caught } \\
\text { the rain. }\end{array}$ \\
\hline Condition B & $13(13.11)$ & $4(4)$ & $0(0)$ & $\begin{array}{l}\text { The farmer smiled, the rain fell, the crops grew, and the economy } \\
\text { improved. }\end{array}$ \\
\hline Condition $\mathrm{C}$ & $20(20.61)$ & $8(8)$ & $0(0)$ & $\begin{array}{l}\text { The farmer watched the clouds, the rain drenched the earth, the } \\
\text { crop broke a record, and the economy showed improvement. }\end{array}$ \\
\hline \multicolumn{5}{|c|}{ Five-Proposition Sentences } \\
\hline Condition A & $16(15.72)$ & $5(5)$ & $4(4)$ & $\begin{array}{l}\text { Signalling the trolley, the anxious tourist reached the corner, } \\
\text { paid the fare, and found a seat. }\end{array}$ \\
\hline Condition B & $16(15.72)$ & $S(5)$ & $0(0)$ & $\begin{array}{l}\text { The tourist waited, the trolley approached, the bell rang, the } \\
\text { brakeman signalled, and the passengers boarded. }\end{array}$ \\
\hline Condition $\mathrm{C}$ & $22(21.27)$ & $8(8)$ & $0(0)$ & $\begin{array}{l}\text { The tourist reached the corner, the trolley approached, the } \\
\text { brakeman rang the bell, the passengers boarded, and the pay- } \\
\text { master collected the fare. }\end{array}$ \\
\hline
\end{tabular}

Note-Mean values for all sentences are in parentheses. The words in italics were the cues. They also refer to the concepts shared by all propositions in Condition A. Aside from that sharing, the commas in all sentences separate the words corresponding to the underlying propositions.

sentences for each subject were grouped into three equal-sized lists $(\mathrm{N}=12$ sentences per list). Within each half of each list, equal numbers of sentences from the various conditions and lengths were represented. One filler sentence was added to the beginning and the end of each list in order to reduce primacy and recency effects. Counterbalancing across subjects was done by using Latin squares to insure that each sentence set was used for each condition, although each subject was presented only one sentence from each set. The lists were balanced such that each list was presented first, second, or third an equal number of times across subjects.

Procedure. Students were tested individually or in groups of up to six people. The immediate and delayed testing conditions were in separate experimental sessions. Within each session, assignment of subjects to type of recall (free or cued) was determined through random distribution of recall booklets.

Oral instructions to all subjects explained the task as an exercise on memory for sentences. They were told that the sentences would appear individually, projected on the wall via an overhead projector. Subjects were also informed that the sentences would appear for a fixed amount of time. (Determination of the time is explained below.) Subjects in the immediate test conditions were told that they would recall each sentence immediately after its presentation. Subjects in the delayed test conditions were told that their memory test would occur after a large number of sentences had been presented.

After these general instructions, students opened their recall booklets and read further instructions. The written directions told students to recall verbatim, if possible, but encouraged them to write anything they remembered: words, synonyms, phrases, or incomplete sentences. Additionally, students in the cued recall groups read that a word would appear at the top of each recall page and that the words should help to remember the sentences. All learners then proceeded through four practice sentences in a manner that corresponded to their exact testing conditions.

Subjects who received immediate tests recalled after each sentence was presented. Subjects who received delayed tests recalled after each of three blocks of 14 sentences (12 experimental and 2 fillers). The order of the cues for the delayed cued recall subjects was identical to the order of the corresponding sentences. All subjects received a short rest period after two-thirds of the experiment had been completed.

Timing. Presentation of the sentences and recall intervals was controlled by an audiotape. In the immediate conditions, subjects read each sentence for a predetermined amount of time, followed by a $42 \mathrm{sec}$ interval for recall. In each block of the delayed conditions, 14 sentences appeared sequentially, separated by 5 -sec intervals; immediately after each block, subjects were given $8.5 \mathrm{~min}$ for recall.

The time allowed for reading the sentences was determined by a procedure used before in similar experiments (Kintsch, Kozminsky, Streby, McKoon, \& Keenan, 1975; Manelis \& Yekovich, 1976). Each of nine subjects (different from those tested in the main experiment) read a group of 36 sentences and gave immediate recall to each one. The sentences were presented individually in a tachistoscope, and subjects were told to "read the sentence as quickly as you can, but take time to comprehend its meaning, because you will receive a memory test on each item." Subjects controlled the initiation and termination of each sentence. Reading time was measured with the use of a Hunter Klockounter. The resulting reading times were averaged for each sentence length, collapsing across Conditions $\mathrm{A}, \mathrm{B}$, and $\mathrm{C}$. The presentation times for the main experiment were then set at $50 \%$ of the mean reading time for each sentence length. These times were $5 \mathrm{sec}$ for four-proposition sentences and $6 \mathrm{sec}$ for five-proposition sentences.

Subjects. The subjects were 108 undergraduate volunteers from Arizona State University. They received course credit for their participation.

\section{Results and Discussion}

The subjects' protocols were scored for gist recall. The data were examined in two ways: in terms of the proportion of propositions recalled correctly and in terms of the number of sentences from which at least one proposition was recalled. The number of such atleast-partial recalls was taken as a measure of successful accesses to sentences in memory. The statistical analyses utilized subjects and items separately as random effects. The traditional way to demonstrate statistical significance is by means of subject analysis. Consideration of variability across items has also been discussed (Clark, 1973; Wike \& Church, 1976). We will present $F$ values from subject analyses $\left(F_{1}\right)$ as our primary evidence, 
supplemented by $F$ values from item analyses $\left(F_{2}\right)$ as checks on the generality of the results across items. For the conclusions that we draw, $F$ values were significant in both types of analyses $(p<.05)$.

Propositions recalled. Table 2 presents the mean proportions of propositions recalled. Analyses on arcsin transformations of the data yielded several effects of only incidental interest, and thus we will simply report those results without elaboration. Every main effect was significant: time of test (immediate $>$ delay), type of recall (cued $>$ free), sentence length (four $>$ five), and sentence condition. Further, four ancillary two-way interactions occurred: Time of Test by Type of Recall, Time of Test by Sentence Condition, Type of Recall by Sentence Condition, and Sentence Condition by Sentence Length.

The access hypothesis states that recall differences between Sentence Conditions A and B should vary as a function of accessibility of their associated memory structures. Within the present context, then, observed A-B differences should vary according to the testing combination. This effect was represented overall by the triple interaction of Time of Test by Type of Recall by Sentence Condition $\left[\mathrm{F}_{1}(2,208)=5.80, \mathrm{p}<.005\right.$, $\left.\mathrm{F}_{2}(2,68)=7.49, \mathrm{p}<.005\right]$. To determine the validity of the access idea, individual comparisons were made among sentence conditions within each testing condition. Under testing conditions in which access to sentences in memory was likely, integrated sentences were recalled better than nonintegrated ones [for immediate free recall, the A-B contrast produced $F_{1}(1,208)=7.84$, $\mathrm{p}<.01, \mathrm{~F}_{2}(1,68)=8.06, \mathrm{p}<.01 ;$ for immediate cued recall, $\quad F_{1}(1,208)=9.19, \quad p<.005, \quad F_{2}(1,68)=9.44$, $p<.005]$. Similarly, when access was aided by recall prompts, the expected facilitation of A over B occurred [for delayed cued recall, $F_{1}(1,208)=19.46, p<.001$, $\left.\mathrm{F}_{2}(1,68)=20, \mathrm{p}<.001\right]$. In contrast, when access probability was low, as in delayed recall, integrated memory structures were remembered slightly worse than nonintegrated ones; the means were .186 and .200 for Conditions $\mathrm{A}$ and $\mathrm{B}$, respectively. This difference was not statistically reliable (both $F s<1$ ). Nevertheless, these results reaffirm our earlier results (Manelis \&

Table 2

Mean Proportion of Propositions Recalled as a Function of Experimental Condition, Experiment 1

Sentence Length

\begin{tabular}{lccccccc} 
& \multicolumn{3}{c}{ Four Propositions } & & \multicolumn{3}{c}{ Five Propositions } \\
\cline { 2 - 4 } \cline { 5 - 7 } & $\mathrm{A}$ & $\mathrm{B}$ & $\mathrm{C}$ & $\mathrm{A}$ & $\mathrm{B}$ & $\mathrm{C}$ \\
\hline Free & .791 & .773 & .408 & .659 & .571 & .397 \\
Cued & .772 & .749 & .400 & .656 & .562 & .407 \\
& \multicolumn{5}{c}{ Delayed } \\
Free & .235 & .245 & .070 & .136 & .155 & .088 \\
Cued & .417 & .332 & .105 & .299 & .214 & .165 \\
\hline
\end{tabular}

Yekovich, 1976), and the triple interaction suggests strongly that differential accessibility to the representations in memory contributes to this recali phenomenon.

Additional contrasts showed that Condition $\mathrm{C}$ sentences were recalled least well under all testing conditions ( $p<.001$ for all contrasts). For immediate recall, the results provided a replication of the Manelis and Yekovich (1976) experiments. The disappointing result was the severe recall decrement of Condition $C$ sentences under delayed free testing. Apparently, the extra arguments (and/or extra words) put these sentences at a severe disadvantage during acquisition. It is probable that the subjects were not able to process these sentences because of the reading time restriction. This processing difficulty effectively destroyed any potential access advantage of the extra propositional arguments.

Because our access idea was motivated primarily by Conditions $A$ and $B$, and since Condition $C$ sentences may have contributed noise to the triple interaction, we reanalyzed the data, removing Condition $C$. This reanalysis did not alter the major pattern of results just described. The triple interaction occurred as predicted $\left[F_{1}(1,104)=6.41, p<.02, F_{2}(1,34)=7.16, p<.02\right]$, and the contrasts produced effects comparable to those in the larger analysis.

Sentences accessed. The mean proportions of sentences accessed are given in Table 3. Comparison between Tables 2 and 3 reveals similar patterns in the two types of data. The analyses confirmed these similarities statis tically, except for a few expected deviations. First, in contrast to propositional recall, the number of sentences accessed did not produce a main effect for sentence length (both $\mathrm{Fs}<1$ ). That is, fragments from fiveproposition sentences occurred as often in recall as did four-proposition fragments. Second, the triple interaction among time of test, type of recall, and sentence condition was statistically reliable $\left[F_{1}(2,208)=9.08, p<.001\right.$, $\left.F_{2}(2,68)=10.62, p<.001\right]$, but it took a slightly different form from that in the previous analyses. Here, the immediate conditions led to such high sentence access rates that a ceiling effect obscured the A-B differences. This result supports our belief that in immediate recall, subjects should have little trouble accessing memory for any sentence, regardless of its type. The important contrasts concerned the delayed testing conditions, and these results mirrored the previous analyses. When recall prompts were available, delayed recall showed an advantage for access of Condition A sentences $\left[F_{1}(1,208)=\right.$ $\left.34.85, \mathrm{p}<.001, \mathrm{~F}_{2}(1,68)=49.42, \mathrm{p}<.001\right]$. However, without cues, accesses slightly favored $B$ sentences over A sentences (the mean proportions were .42 and .38 for $B$ and A sentences, respectively). Again, this difference was not significant $\left(\mathrm{F}_{1}=1.95, \mathrm{~F}_{2}=2.67\right)$, but it confirmed our earlier results.

Again, we completed two sets of analyses: one set, described above, in which Condition $\mathrm{C}$ was included, and one from which it was excluded. The two sets of analyses 
Table 3

Mean Proportion of Sentences Accessed, Experiment 1

Sentence Length

\begin{tabular}{lccccccc} 
& \multicolumn{3}{c}{ Four Propositions } & \multicolumn{3}{c}{ Five Propositions } \\
\cline { 2 - 4 } \cline { 5 - 7 } & $\mathrm{A}$ & $\mathrm{B}$ & $\mathrm{C}$ & $\mathrm{A}$ & $\mathrm{B}$ & $\mathrm{C}$ \\
\hline & \multicolumn{7}{c}{ Immediate } \\
Free & .993 & .980 & .845 & 1.000 & .987 & .913 \\
Cued & .981 & .993 & .863 & .993 & .975 & .900 \\
& \multicolumn{7}{c}{ Delayed } \\
Free & .413 & .427 & .197 & .352 & .413 & .283 \\
Cued & .722 & .592 & .327 & .698 & .518 & .450 \\
\hline
\end{tabular}

produced identical patterns of results [in particular, for the three-way interaction of sentence condition, type of test, and time of test, $F_{1}(1,104)=19.45, p<.001$, $\left.\mathrm{F}_{2}(1,34)=19.48, \mathrm{p}<.001\right]$.

In short, Experiment 1 supported our interpretation of memory for integrated and nonintegrated sentences. Clearly, integrated memory structures led to better recall than nonintegrated ones under conditions of high probability of access. However, integrated structures were reduced to a slight disadvantage when access probability was low. This condition of low access thus represents an interesting and novel result, one that was replicated and extended in Experiment 2.

\section{EXPERIMENT 2}

Although the accessibility notion provides a parsimonious explanation for the observed advantage of Condition B over Condition A in delayed free recall, this result is highly counterintuitive. It is inconsistent with the generally supported conclusion that items of information are remembered better if they are related to each other than if they are not. Originally, before we had thought of the accessibility explanation, we found it disturbing (and so did a reviewer of our earlier paper) that the superiority of Condition A over Condition B occurred only in immediate recall and was eliminated or even reversed at a delay. Thus, we felt it necessary to attempt a replication of the counterintuitive result before we could feel confident about interpreting it in more detail according to recent theory.

In the present experiment, subjects were presented sentences in Conditions A and B only; the recall test was always free, never cued, and it occurred only after a block of sentences, corresponding to the delayed testing in Experiment 1.

Additionally, Experiment 2 investigated whether encoding conditions, rather than retrieval conditions, could alter the accessibility of integrated and nonintegrated memory structures. Several researchers have shown that people can be encouraged to selectively attend to critical features of a text by highlighting certain salient material (see the review by Anderson, 1970). Such highlighting influences the encoding strategies that subjects use during acquisition and, consequently, affect what can be recalled. In this experiment, we incorporated a highlighting technique into the presentation format of the sentences, on the assumption that the highlighted words would have an especially good chance of being retrieved in free recall. The presentation of each sentence either conformed to that in Experiment 1 ("standard format"), or the word corresponding to the cue in the prior experiment was underlined ("underlined format"). Just as the slight superiority of Condition B over Condition A was reversed for cued recall in Experiment 1, we thought that underlining the cue words (and encouraging subjects to attend to them) might moderate any superiority of $\mathrm{B}$ over $\mathrm{A}$ in the present experiment, even though here the underlined words were never presented as cues at the time of the tests. Thus, our reasoning predicts an interaction analogous to the one in the first experiment, only here the factors should include sentence type and presentation format. Obtaining this result would support our interpretation of the counterintuitive findings in Experiment 1.

\section{Method}

Design. Four independent variables were combined factorially . The two within-subjects variables were sentence condition $(A, B)$ and sentence length (four propositions, five propositions). The two between-subjects variables were block size (4 or 16 sentence presentations before a test) and presentation format (standard, underlined).

Materials and counterbalancing. In order to accommodate the counterbalancing, two randomly selected four-proposition sentence sets and two randomly selected five-proposition sentence sets were discarded from the materials of the prior experiment. The remaining 32 sets were divided into two groups. Each group contained eight four-proposition sets and eight five-proposition sets. Half of the subjects were shown the A sentences from the first group and the B sentences from the second group; the other half of the subjects were shown the B sentences from the first group and the $A$ sentences from the second group.

The order of the sentences shown to any one group of subjects was randomized with the constraint that each successive block of four sentences should contain two of each length (four or five propositions) and two of each condition (A or B). Half of the subjects received one such block of 4 sentences before each of eight recall tests, and the other half of the subjects received four blocks (16 sentences) before each of two tests. The order of the sentences was the same for the 4-block subjects as for the 16 -block subjects, although the preestablished orders were presented in reverse half of the time. Orthogonal to all of the above, for half of the subjects, the sentences were presented in standard format and, for the other half, in underlined format.

Procedure. As in the prior experiment, all materials were presented visually via an overhead projector, and the timing of presentations and test intervals was accomplished by prerecorded signals on an audiotape. Each sentence was displayed for $6 \mathrm{sec}$, and the recall intervals were $2.5 \mathrm{~min}$ after 4 -sentence blocks and $7 \mathrm{~min}$ after 16 -sentence blocks.

At the beginning of the experiment, all subjects were presented a block of four practice sentences and a practice recall test. The practice included the same types of sentences that were presented in the main part of the experiment. Subjects who were shown the sentences in the underlined format were told to pay special attention to the underlined words because those words could help them to recall. The instructions for the recall tests were to write as much as possible from the previous block of sentences, to use synonyms if exact words could not be remem- 
bered, to guess if unsure, and to write the remembered information in any order. The entire procedure of presenting blocks of sentences interspersed with recall tests took $30-45 \mathrm{~min}$. All subjects returned 1 day after this procedure and were given an unexpected free recall test on all of the sentences from the previous day.

Subjects. Ninety-six psychology students served as subjects in exchange for payment or for extra credit in their classes. Subjects were tested individually or in groups of up to six.

\section{Results and Discussion}

Subjects' protocols were scored for gist recall. Again, the data were examined in terms of the proportion of propositions recalled correctly and in terms of the number of successful accesses to sentences in memory. Analyses of variance were computed on sentences accessed and on proportional recall (arcsin transformations) for the 1st and 2 nd days of testing. The independent variables in these analyses were sentence condition $(A, B)$, sentence length (four, five propositions), block size $(4,16$ sentences), and presentation format (standard, underlined). We will describe the statistically significant results of the analyses, as well as other comparisons involving Conditions $A$ and $B$. There are three points to be made. First, there were some significant results of only incidental interest. Second, there was a key interaction very similar to the triple interaction in Experiment 1 . Third, the average performance in Condition $B$ was better than the average performance in Condition $A$.

First, as might be expected, there were significant effects of block size and sentence length. For Day 1 of the experiment, there were significant effects $(p<.05)$ of block size and sentence length on both proportional recall and sentence accesses, with poorer performance for the longer blocks and sentences. For Day 2, there were significant effects of block size and sentence length on proportional recall but not on sentence accesses.

Second, on Day 1, there was an interaction between presentation format and sentence condition (A vs. B) for sentence accesses. This interaction is shown in Table 4 . With the standard presentation format, more B than $A$ sentences were accessed, but with the underlined format, there were more accesses for $A$ than $B$ [for the interaction terms in the analyses of variance, $F_{1}(1,88)=$ $\left.5.48, \mathrm{p}<.025, \mathrm{~F}_{2}(1,16)=4.20, \mathrm{p}<.057\right]$. Although the difference between $A$ and $B$ for the standard format was not significant by contrasts within the analyses of variance, most of the subjects and most of the items did show better performance with B (both ps $<.05$ by two-

Table 4

Sentence Accesses in Experiment 2

\begin{tabular}{ccc}
\hline & \multicolumn{2}{c}{ Sentence Condition } \\
\cline { 2 - 3 } $\begin{array}{c}\text { Presentation } \\
\text { Format }\end{array}$ & $\mathrm{A}$ & $\mathrm{B}$ \\
\hline Standard & .609 & .654 \\
Underlined & .681 & .654 \\
\hline
\end{tabular}

Note-The figures are proportions of the total possible accesses on Day 1.
Table 5

Comparisons of Conditions A and B in Experiment 2

\begin{tabular}{cccc}
\hline \multirow{2}{*}{$\begin{array}{c}\text { Dependent } \\
\text { Measure }\end{array}$} & Day & & \multicolumn{2}{c}{ Sentence Condition } \\
\cline { 4 - 5 } & 1 & .645 & $\mathrm{~B}$ \\
\hline \multirow{2}{*}{ Sentence Accesses } & 2 & .374 & .654 \\
& 1 & .295 & .398 \\
Proportional Recall & 2 & .137 & .158 \\
\hline
\end{tabular}

tailed sign tests). The finding of statistical significance by two-tailed sign tests despite the lack of significance for the contrasts within the analysis of variance is rather unusual. The discrepancy can be interpreted by the difference in statistical models: If an effect is inherently variable in magnitude even though it is consistent in direction, then a sign test is more likely to detect it. Thus, any advantage of Condition B over Condition A must be considered variable in magnitude from subject to subject and from item to item. Further, the fact that it has been observed in the present experiment and in Experiment 1 as well as in our prior work (Manelis \& Yekovich, 1976) makes it difficult for us to dismiss it. At any rate, aside from the question of individual simple effects, the pattern of results parallels the key interaction found in Experiment 1. In that experiment, it was found that at delayed testing, free recall of $B$ sentences was better than free recall of A sentences, but cued recall of A sentences was better than cued recall of $B$ sentences. In the present experiment there was no cued testing, but highlighting the cue words at the time of the study was enough to offset any superiority of Condition B over Condition A. The most obvious interpretation is that highlighting allowed subjects to retain and subsequently use the critical words as cues on their own. Thus, highlighting had the effect of formally prompting subjects to attend to critical text features during acquisition of the material (Anderson, 1970).

The third point to be made about the results of this experiment is that, when averaged over all other independent variables, performance in Condition $B$ was slightly better than performance in Condition A, just as in the corresponding test in Experiment 1: delayed free recall. This superiority of B over A is shown in Table 5 for both the 1 st and 2 nd days of the experiment, as well as for both proportional recall and sentences accessed.

\section{GENERAL DISCUSSION}

The results observed in this study can be interpreted in a way that parallels theory on lag effects in list learning. In that area, there is an interaction between two factors: the lag between two presentations of an item in a list and the retention interval between the second presentation and a test. The form of this inter. action is that, for long retention intervals, longer presentation lag produced better recall, whereas for short 
retention intervals, longer lag produces poorer recall. Crowder (1976, pp. 291-309) discusses this interaction as a criterion for evaluating and synthesizing theories to explain lag effects. As Crowder points out, the interaction is not always as simple as stated above because sometimes lag effects are nonmonotonic (e.g., Glenberg, 1976). However, the simple form of the interaction is accurate in many cases, and within a limited range of lags and retention intervals, the simple form is compatible with current theory (e.g., Crowder, 1976; Glenberg, 1976; Landauer, 1975). We believe that our results parallel this interaction and can be interpreted by using a parallel kind of theory.

Lag effect theory is concerned with relationships among the memory traces for item repetitions. Instead of traces for item repetitions, however, our present concern is with memory traces for propositions. The trace for a proposition can be considered a set of points in a space, corresponding to the concepts that the proposition includes. In Figure 1, each proposition is thus shown as a circle (cf. Glenberg, 1976, p. 6.). For each Condition A sentence, the propositions share a concept, represented in the figure by an intersection. (For simplicity, only two propositions are shown.) For each Condition B sentence, the explicit propositions are not directly connected and are represented as disjoint. However, there is some probability that in an attempt to comprehend a B sentence, subjects will add their own propositions that will connect those conveyed in the sentence more explicitly. Such added propositions have been known in the literature as one type of "inference," and there is evidence that these inferences are made (Clark, 1977; Kintsch, 1974, 1978). In terms of the memory space, we represent an inference as an additional set intersecting the explicit propositions, thus providing a "bridge" over the conceptual gap (Clark, 1977).

Following Glenberg (1976) and related theory (Crowder, 1976), we assume that in an immediate free recall test, because the material is so recent, subjects have a very high probability of accessing some element of the memory trace. This initial access would activate

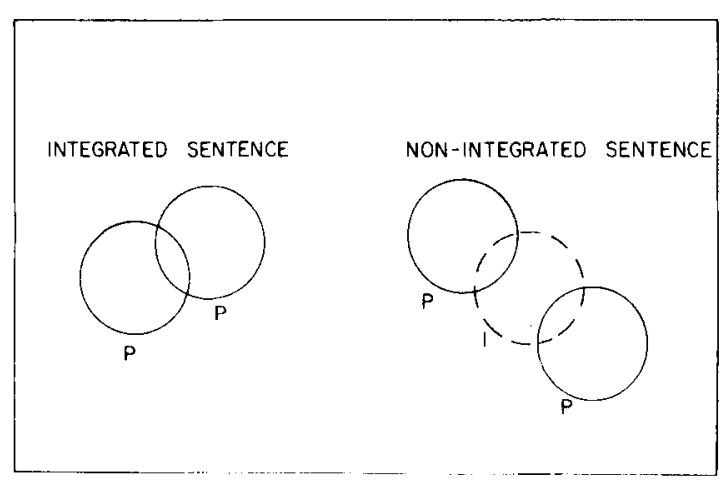

Figure 1. Memory representations for integrated and nonintegrated sentences. $P$ denotes a proposition and 1 , an inference. some element within one of the proposition sets represented in Figure 1. Assuming that subsequent retrieval of other parts of the memory trace will be difficult as a function of the distance between them and the initial access point, one would expect more propositions to be recalled from A sentences than from B sentences. This was the result observed in Experiment 1 when a free recall test was given immediately following presentation (also Manelis \& Yekovich, 1976). When free recall was delayed, however (Experiments 1 and 2), recall for Condition B was slightly better than for Condition A. Again, following lag effect theory, we assume that a retrieval attempt after a long retention interval may activate any point throughout the memory space; initial access to the propositions studied is no longer certain. Furthermore, following the arguments explicated by Glenberg (1976) and Landauer (1975), because the traces for B propositions do not overlap and are more dispersed than the traces for A propositions, successful access of a B proposition is more likely, thus producing better recall than in Condition A. Thus, for free recall, the interaction between retention interval and sentence type can be explained in a way that parallels the interaction between retention interval and presentation lag.

It is reasonable, however, for the interaction to be altered when recall is cued (cf. Glenberg, 1977). In terms of the memory representation shown in Figure 1, we assume that providing a cue will activate part of the memory trace, regardless of whether the test is immediate or at a delayed retention interval. Thus, the reasoning for immediate free recall (as described above) applies to cued recall. Therefore, cued recall in Condition A should exceed cued recall in Condition B at both immediate and delayed testing, as observed in Experiment 1.

This explanation of our results has the advantage of integrating memory for prose with more traditional listlearning research. Further, based on our explanation, there are two additional predictions that can be made. One is that the superiority of Condition B over Condition $A$ in delayed recall ought to be enhanced as the number of propositions per sentence increases. This would be expected because the access advantage for B sentences would increase with more propositions. In fact, the sentence access analysis in Experiment 1 showed some support for this idea (see Table 3 ). In delayed free recall, the advantage of Type $B$ sentences over Type A sentences was .014 for four-proposition sentences and .061 for the five-proposition sentences. (This difference is not statistically significant, but the variation in sentence length here is only between four and five propositions.) $\mathrm{A}$ second prediction is that the recall superiority of Condition B over Condition A should be enhanced by encouraging subjects to elaborate or supply inferences. To the extent that inferences occur, they should benefit Condition B more than Condition A because the $\mathrm{A}$ propositions are already connected. Encouraging inferences would provide still further access 
advantage to $B$ sentences in the memory space and, once an initial access point is reached, would provide new retrieval paths between the otherwise isolated propositions.

Finally, we should point out two limitations of the present experiments. One is that the variation between Conditions $\mathrm{A}$ and $\mathrm{B}$ represents only a certain range of integration. In list-learning research, performance varies considerably over a broad range of lags, and the lag functions are not always monotonic. With sentence materials, it may also be that the nature of the effects varies considerably over a range of integration broader than that represented in our study. In this regard, our choice of sentence conditions may have been fortuitous in allowing us to see that nonintegrated sentences can provide certain access advantages.

A second limitation of the present experiments is that they dealt with simple fact recall and did not include higher order comprehension tasks. It may be that tasks requiring subjects to utilize relations among individual ideas benefit from integrated text materials, even though nonintegrated or Type B text can be relatively beneficial for simple recall (cf. Hayes-Roth \& Walker, 1979).

\section{REFERENCES}

Anderson, R. C. The control of student mediating processes during verbal learning and instruction. Review of Educational Research, 1970, 40, 349-370.

Clark, H. H. The language-as-fixed-effect fallacy: A critique of language statistics in psychological research. Journal of Verbal Learning and Verbal Behavior, 1973, 12, 335-359.

CLARK, H. H. Inferences in comprehension. In D. LaBerge \& S. J. Samuels (Eds.), Basic processes in reading: Perception and comprehension. Hillsdale, N.J: Erlbaum, 1977.
Crowder, R. G. Principles of learning and memory. Hillsdale, N.J: Erlbaum, 1976.

GlenberG, A. M. Monotonic and nonmonotonic lag effects in paired-associate and recognition memory paradigms. Journal of Verbal Learning and Verbal Behavior, 1976, 15, 1-15.

Glenberg, A. M. Influences of retrieval processes on the spacing effect in free recall. Journal of Experimental Psychology: Human Learning and Memory, 1977, 3, 282-294.

Glenberg, A. M. Component-levels theory of the effects of spacing of repetitions on recall and recognition. Memory \& Cognition, 1979, 7, 95-112.

HAyes-Roth, B., \& ThoRndyke, P. W. Integration of knowledge from text. Journal of Verbal Learning and Verbal Behavior, 1979. 18, 91-108.

Hayes-Roth, B., \& Walker, C. H. Configural effects in human memory. Cognitive Science, 1979, 3, 119-140.

$\mathrm{K}_{\mathrm{INTSCH}}, \mathrm{W}$. The representation of meaning in memory. Hillsdale, N.J: Erlbaum, 1974.

Kinrsch, W. Comprehension and memory for text. In W. K. Estes (Ed.), Handbook of learning and cognitive processes (Vol. 6). Hillsdale, N.J: Erlbaum, 1978.

Kintsch, W., Kozminsky, E., Streby, W. J., McKoon, G., \& Keenan, J. M. Comprehension and recall of text as a function of content variables. Journal of Verbal Learning and Verbal Behavior, 1975, 14, 196-214.

Landauer, T. K. Memory without organization: Properties of a model with random storage and undirected retrieval. Cognitive Psychology, 1975, 7, 495-531.

MANElis, L., \& Yekovich, F. R. Repetitions of propositional arguments in sentences. Journal of Verbal Learning and Verbal Behavior, 1976, 15, 301-312.

Moeser, S. D. The role of experimental design in investigations of the fan effect. Journal of Experimental Psychology: Human Learning and Memory, 1979, 5, 125-134.

Thorndyke, P. W. Cognitive structures in comprehension and memory of narrative discourse. Cognitive Psychology, 1977, 9. 77-110.

Wike, E. L., \& Church, J. D. Comments on Clark's “The language-as-fixed-effect fallacy." Journal of Verbal Learning and Verbal Behavior, 1976, 15, 249-255.

(Received for publication July 6, 1979; revision accepted December 10,1979.) 